\title{
Complex dynamics in an SIS epidemic model with nonlinear incidence
}

\author{
Ruixia Yuan ${ }^{1}$, Zhidong Teng ${ }^{2}$ and Jinhui $\mathrm{Li}^{1 *}$
}

"Correspondence:

lijinhui2050@163.com

'School of Mathematics and

Statistics, Central China Normal

University, Wuhan, China

Full list of author information is

available at the end of the article

\begin{abstract}
We study an epidemic model with nonlinear incidence rate, describing the saturated mass action and the psychological effect of certain serious diseases on the community. Firstly, the existence and local stability of disease-free and endemic equilibria are investigated. Then we prove the occurrence of backward bifurcations, saddle-node bifurcations, Hopf bifurcations and cusp type Bogdanov-Takens bifurcations of codimension 3. Finally, numerical simulations, including one limit cycle, two limit cycles, an unstable homoclinic loop and many other phase portraits are presented. These results show that the psychological effect of diseases and the behavior change of the susceptible individuals may affect the final spread level of an epidemic.
\end{abstract}

Keywords: Epidemic; Nonlinear incidence rate; Saturated mass action; Psychological effect; Bifurcation

\section{Introduction}

In the well-known SIS epidemic model, the population is always separated into two compartments, susceptible and infective individuals. In most SIS epidemic models (see Anderson and May [1]), the incidence takes the mass-action form with bilinear interactions. However, in a practical application, to describe the transmission process more realistically, it is necessary to introduce the nonlinear contact rates [2].

Actually, various forms of nonlinear incidence rates have been proposed recently [310]. For example, in order to incorporate the effect of behavioral changes, Liu, Levin, and Iwasa [6] used a nonlinear incidence rate of the form

$$
g(I) S=\frac{\kappa I^{l} S}{1+\alpha I^{h}},
$$

where $\kappa I^{l}$ represents the infection force of the disease, $1 /\left(1+\alpha I^{h}\right)$ is a description of the suppression effect from the behavioral change of susceptible individuals when the infective population increases. $\iota, h$ and $\kappa$ are all positive constants, and $\alpha$ is a nonnegative constant. See also Hethcote and van den Driessche [7], Moghadas [8] and Alexander and Moghadas $[9,10]$, etc.

To describe the effects of psychology effect caused by protection measures and intervention policies, etc., when a serious disease arouses widespread horror, in [11], Ruan

(c) The Author(s) 2019. This article is distributed under the terms of the Creative Commons Attribution 4.0 International License (http://creativecommons.org/licenses/by/4.0/), which permits unrestricted use, distribution, and reproduction in any medium, provided you give appropriate credit to the original author(s) and the source, provide a link to the Creative Commons license, and indicate if changes were made. 
(a)

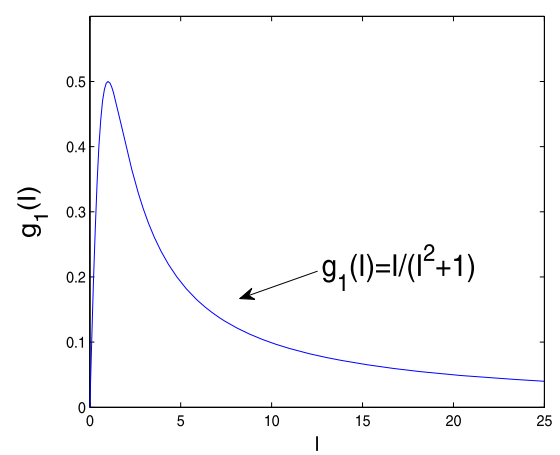

(b)

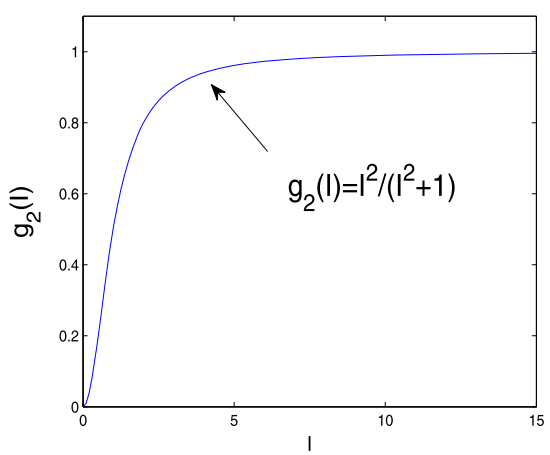

(c)

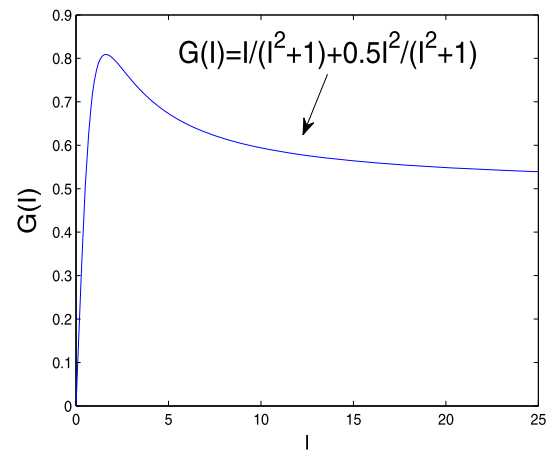

Figure 1 Graphs of different incidence rate functions. (a) Non-monotone incidence $g_{1}(I)=\frac{\kappa l}{1+\alpha \alpha^{2}}$.

(b) Nonlinear incidence $g_{2}(l)=\frac{\kappa l^{2}}{1+\left.\alpha\right|^{2}}$. (c) Nonlinear and non-monotone incidence rate $G(l)=\frac{a l^{2}}{l^{2}+c}+\frac{b l}{l^{2}+c}$

discussed a specific infection force

$$
g_{1}(I)=\frac{\kappa I}{1+\alpha I^{2}}
$$

see Fig. 1(a). Obviously, $g_{1}(I)$ is increasing with small $I$ and decreasing with large $I$, that is, $g_{1}(I)$ is non-monotone. It can be used to interpret the "psychological" effect: for a very large number of infective individuals, the infection force may decrease as the number of infective individuals increases, since a large number of infectives may lead to the reducing of the number of contacts per unit time. For example, in 2003, the epidemic outbreak of severe acute respiratory syndrome (SARS) had such psychological effects on the general public (see [12]), and aggressive measures and policies had been taken, such as border screening, mask wearing, quarantine, isolation, etc. One showed that either the number of infective individuals tends to zero as time evolves or the disease persists.

Furthermore, Li, Zhao and Zhu (see [13]) studied the following SIS model, which describes behavior change effect of susceptible individual when infectious population increases:

$$
\left\{\begin{array}{l}
\frac{d S}{d t}=\Lambda-d_{0} S-g_{2}(I) S+\delta I \\
\frac{d I}{d t}=g_{2}(I) S-\left(d_{0}+d^{\prime}+\delta\right) I
\end{array}\right.
$$


where

$$
g_{2}(I)=\frac{\alpha I^{2}}{1+\beta_{0} I^{2}}
$$

see Fig. 1(b). By the qualitative and bifurcation analyses, they showed that the maximal multiplicity of weak focus is 2 , and proved that the model can undergo a Bogdanov-Takens bifurcation of codimension 2 . These results illustrate that the behavior change of the susceptible individuals may affect the final spread level of an epidemic.

Actually, both the effect of psychology and the behavior change of susceptible individuals have influence on the transmission of the disease. Thus, motivated by the above research, we consider a nonlinear incidence rate of a SIS model as follows:

$$
\left\{\begin{array}{l}
\frac{d S}{d t}=\Lambda-S G(I)-d S+\sigma I, \\
\frac{d I}{d t}=S G(I)-(d+\mu+\sigma) I,
\end{array}\right.
$$

where

$$
G(I)=\frac{a I^{2}}{c+I^{2}}+\frac{b I}{c+I^{2}}
$$

see Fig. 1(c), which can describe the effect of psychology and behavior change of susceptible individuals. $S$ and $I$ represent the number of susceptible individuals and infected individuals, respectively. $\Lambda$ is the recruitment rate of population, $d$ is the natural death, $\mu$ is the disease-induced death rate, and $\sigma$ represents the recovered rate, $a, b$ and $c$ are all positive constants.

The organization of this paper is as follows. In Sect. 2, we analyze the existence of the equilibria and local stability of the equilibria. In Sect. 3, we study the existence of Hopf bifurcation around the positive equilibrium at the critical value under the conditions of $R_{0}<1$ and $R_{0}>1$. We also show that these positive equilibria can be weak focus for some parameter values and a cusp type of Bogdanov-Takens bifurcation of codimension 3. In Sect. 4, we give some brief discussions.

\section{Types and stability of the equilibria}

Firstly, we make scalings: $\left(\Lambda^{\prime}, a^{\prime}, d^{\prime}, \sigma^{\prime}, c^{\prime}\right)=\left(\frac{\Lambda}{b}, \frac{a}{d+\mu+\sigma}, \frac{d}{d+\mu+\sigma}, \frac{\sigma}{d+\mu+\sigma}, \frac{c(d+\mu+\sigma)^{2}}{b^{2}}\right)$, and $(x, y$, $\tau)=\left(\frac{d+\mu+\sigma}{b} S, \frac{d+\mu+\sigma}{b} I,(d+\mu+\sigma) t\right)$. To avoid the abuse of mathematical notation, we still denote $\left(\Lambda^{\prime}, a^{\prime}, d^{\prime}, \sigma^{\prime}, c^{\prime}, \tau\right)$ by $(\Lambda, a, d, \sigma, c, t)$. Then model (1.2) becomes

$$
\left\{\begin{array}{l}
\frac{d x}{d t}=\Lambda-\frac{x y(a y+1)}{y^{2}+c}-d x+\sigma y, \\
\frac{d y}{d t}=\frac{x y(a y+1)}{y^{2}+c}-y,
\end{array}\right.
$$

where $d+\sigma<1$.

Lemma 2.1 The set $D=\left\{(x, y) \mid x \geq 0, y \geq 0, x+y \leq \frac{\Lambda}{d}\right\}$ is an invariant manifold of system (2.1), which is attracting in the first octant of $\mathbb{R}^{2}$. 
Proof Summing up the two equations in (2.1), we can get

$$
\frac{d(x+y)}{d t}=\Lambda-d(x+y)-(1-d-\sigma) y \leq \Lambda-d(x+y) .
$$

Thus, $\lim \sup _{t \rightarrow \infty}(x+y) \leq \frac{\Lambda}{d}$, which implies the conclusion.

Obviously, system (2.1) always has a unique disease-free equilibrium $E_{0}=\left(\frac{\Lambda}{d}, 0\right)$. The positive equilibria of (2.1) can be obtained by solving the following algebraic equations:

$$
\Lambda-\frac{x y(a y+1)}{y^{2}+c}-d x+\sigma y=0, \quad \frac{x y(a y+1)}{y^{2}+c}-y=0,
$$

which yields

$$
(d+a(1-\sigma)) y^{2}+(1-\sigma-a \Lambda) y+d c-\Lambda=0
$$

Denote the basic reproduction number as follows:

$$
R_{0}=\frac{\Lambda}{d c}
$$

And, for convenience, we define the following quantity:

$$
R^{*}=1-\frac{[(1-\sigma)-a \Lambda]^{2}}{4 d c(d+a(1-\sigma))}
$$

Then, computing the discriminant of (2.2), we get

$$
\begin{aligned}
\Delta & =(1-\sigma-a \Lambda)^{2}-4[d+a(1-\sigma)](d c-\Lambda) \\
& =(1-\sigma-a \Lambda)^{2}-4 d c[d+a(1-\sigma)]\left(1-R_{0}\right) \\
& =4 d c[d+a(1-\sigma)]\left(R_{0}-R^{*}\right)
\end{aligned}
$$

which implies that $\Delta>0$ if and only if $R_{0}>R^{*}, \Delta=0$ if and only if $R_{0}=R^{*}$, and that $\Delta<0$ if and only if $R_{0}<R^{*}$. It is clear that $R^{*}<1$ and we can obtain the following theorem.

Theorem 2.2 Model (2.1) always has a disease-free equilibrium $E_{0}$ and the following conclusions hold.

(i) When $R_{0}<1$, we have

(a) if $R_{0}<R^{*}$, then system (2.1) has no positive equilibrium;

(b) if $R_{0}=R^{*}$ and $\Lambda>(1-\sigma) / a$, then system (2.1) has a unique positive equilibrium $E_{1}\left(x_{1}, y_{1}\right)$, where $x_{1}=\frac{y_{1}^{2}+c}{a y_{1}+1}$ and $y_{1}=\frac{a \Lambda-(1-\sigma)}{d+a(1-\sigma)}$;

(c) if $R_{0}>R^{*}$ and $\Lambda>(1-\sigma) / a$, then system (2.1) has two positive equilibria $E_{2}\left(x_{2}, y_{2}\right)$ and $E_{3}\left(x_{3}, y_{3}\right)$, where $x_{k}=\frac{y_{k}^{2}+c}{a y_{k}+1}(k=2,3)$ and $y_{2}=\frac{a \Lambda-(1-\sigma)-\sqrt{\Delta}}{2(d+a(1-\sigma))}$, $y_{3}=\frac{a \Lambda-(1-\sigma)+\sqrt{\Delta}}{2(d+a(1-\sigma))}$

(ii) When $R_{0}=1$ and $\Lambda>(1-\sigma) / a$, then system (2.1) has a unique positive equilibrium $E_{4}\left(x_{4}, y_{4}\right)$, where $x_{4}=\frac{y_{4}^{2}+c}{a y_{4}+1}$ and $y_{4}=\frac{a \Lambda-(1-\sigma)}{d+a(1-\sigma)}$; 
(iii) When $R_{0}>1$, then system (2.1) has a unique positive equilibrium $E_{5}\left(x_{5}, y_{5}\right)$, where

$$
x_{5}=\frac{y_{5}^{2}+c}{a y_{5}+1} \text { and } y_{5}=\frac{a \Lambda-(1-\sigma)+\sqrt{\Delta}}{2(d+a(1-\sigma))} \text {. }
$$

In the following, we discuss the local stability of $E_{k}\left(x_{k}, y_{k}\right)(k=0,1,2,3,4,5)$ and present the corresponding phase portrait. By directly calculating, the Jacobian matrix at equilibrium $E_{k}$ is

$$
J_{k}=\left(\begin{array}{cc}
-d-\frac{y_{k}\left(a y_{k}+1\right)}{y_{k}^{2}+c} & \sigma-\frac{-y_{k}^{2}+2 a c y_{k}+c}{\left(y_{k}^{2}+c\right)^{2}} x_{k} \\
\frac{y_{k}\left(a y_{k}+1\right)}{y_{k}^{2}+c} & -1+\frac{-y_{k}^{2}+2 a c y_{k}+c}{\left(y_{k}^{2}+c\right)^{2}} x_{k}
\end{array}\right) .
$$

We have

$$
\operatorname{tr} J_{k}=\frac{\psi_{k}}{\left(y_{k}^{2}+c\right)\left(a y_{k}+1\right)}, \quad \operatorname{det} J_{k}=\frac{y_{k} \phi_{k}}{\left(y_{k}^{2}+c\right)\left(a y_{k}+1\right)},
$$

where

$$
\begin{aligned}
& \psi_{k}=-a(d+1+a) y_{k}^{3}-(d+2+2 a) y_{k}^{2}-(a c d+1-a c) y_{k}-c d, \\
& \phi_{k}=a(d+a(1-\sigma)) y_{k}^{2}+2(d+a(1-\sigma)) y_{k}+(1-\sigma)-d a c .
\end{aligned}
$$

In addition, after some complicated computations, we get

$$
\phi_{2}=\frac{\sqrt{\Delta}(a(\sqrt{\Delta}-(a \Lambda+1-\sigma))-2 d)}{2(d+a(1-\sigma))}
$$

and

$$
\phi_{k}=\frac{\sqrt{\Delta}(a(\sqrt{\Delta}+(a \Lambda+1-\sigma))+2 d)}{2(d+a(1-\sigma))}>0 \quad(k=3,4,5) .
$$

Theorem 2.3 The disease-free equilibrium $E_{0}$ of system (2.1) is

(i) an attracting node if $R_{0}<1$;

(ii) a hyperbolic saddle if $R_{0}>1$;

(iii) a saddle-node of codimension 1 if $R_{0}=1$ and $2 a-\frac{1-\sigma}{d c} \neq 0$; a repelling node if $R_{0}=1$ and $2 a-\frac{1-\sigma}{d c}=0$.

Proof Obviously, at equilibrium $E_{0}$, we have $\operatorname{det}\left(J_{0}\right)=d\left(1-R_{0}\right)$ and $\operatorname{Tr}\left(J_{0}\right)=-d-\left(1-R_{0}\right)$. Therefore, $E_{0}$ is a stable node if $R_{0}<1$ and a hyperbolic saddle if $R_{0}>1$, and degenerate if $R_{0}=1$.

When $R_{0}=1$, we let $u=x-\frac{\Lambda}{d}, v=y$, then system (2.1) becomes

$$
\left\{\begin{array}{l}
\frac{d u}{d t}=-d u-(1-\sigma) v-\frac{1}{c} u v-2 a v^{2}+\mathcal{O}\left(|(u, v)|^{3}\right) \\
\frac{d v}{d t}=2 a v^{2}+\frac{1}{c} u v+\mathcal{O}\left(|(u, v)|^{3}\right)
\end{array}\right.
$$

Indeed, if $R_{0}=1$, the Jacobian $J_{0}$ is diagonalizable with eigenvalues $\lambda_{1}=-d$ and $\lambda_{2}=0$ and respective eigenvectors $v_{1}=(1,0)$ and $v_{2}=\left(-\frac{1-\sigma}{d}, 1\right)$. By the transformation

$$
\left(\begin{array}{l}
u \\
v
\end{array}\right)=\left(\begin{array}{cc}
1 & -\frac{1-\sigma}{d} \\
0 & 1
\end{array}\right)\left(\begin{array}{l}
x \\
y
\end{array}\right)
$$


system (2.3) can be rewritten as

$$
\left\{\begin{array}{l}
\frac{d x}{d t}=-d x-\left(\frac{1}{c}+\frac{1-\sigma}{d}\right) x y-\left(\frac{1-\sigma}{c d}-2 a+\frac{2 a(1-\sigma)}{d}\right) y^{2}+\mathcal{O}\left(|(x, y)|^{3}\right), \\
\frac{d y}{d t}=\left(2 a-\frac{1-\sigma}{d c}\right) y^{2}+\frac{1}{c} x y+\mathcal{O}\left(|(x, y)|^{3}\right) .
\end{array}\right.
$$

If $2 a-\frac{1-\sigma}{d c} \neq 0$, according to the calculation of center manifold [14], we know that the center manifold $x=h(y)$ of (2.3) begins with quadratic term of $y$. In addition, from the second equation of (2.3), we can easily see that the equation restricted to the center manifold is as follows:

$$
\frac{d y}{d t}=\left(2 a-\frac{1-\sigma}{d c}\right) y^{2}+\mathcal{O}\left(y^{3}\right)
$$

By applying Theorem 7.1 in Zhang et al. [14], $E_{0}$ is a saddle-node.

If $2 a-\frac{1-\sigma}{d c}=0$, then the center manifold turns into

$$
\frac{d y}{d t}=\frac{1}{c} y^{3}+\mathcal{O}\left(y^{4}\right)
$$

Since $\frac{1}{c}>0$ and the first nonzero item is uneven. Thus, the equilibrium $E_{0}$ is a repelling node, according to Theorem 7.1 in Zhang et al. [14].

From the expression of $\psi_{1}$ and $\phi_{1}$, we can see that one of the eigenvalues of the characteristic matrix of $E_{1}$ is zero and the other is nonzero if $\psi_{1} \neq 0$. The type of $E_{1}$ can be directed proved by checking the conditions in Zhang et al. ([14], Theorems 7.1-7.3). So, we have the following results.

Theorem 2.4 If $R_{0}=R^{*}$, then system (2.1) has a unique positive equilibrium $E_{1}$. More precisely,

(a) if $\psi_{1} \neq 0$, then $E_{1}$ is a saddle-node;

(b) if $\psi_{1}=0$, then $E_{1}$ is a cusp.

Theorem 2.5 Suppose that $R^{*}<R_{0}<1$ and $a \Lambda>1-\sigma$, then system (2.1) has two positive equilibria $E_{2}$ and $E_{3}$, and equilibrium $E_{2}$ is a hyperbolic saddle for all permissible choices of the parameters, equilibrium $E_{3}$ is not degenerate. Moreover,

(i) $E_{3}$ is a stable focus or node if $\psi_{3}<0$;

(ii) $E_{3}$ is a weak focus or center if $\psi_{3}=0$;

(iii) $E_{3}$ is an unstable focus or node if $\psi_{3}>0$.

Proof Note that $\phi_{2}$ is less than zero since $\Delta<(a \Lambda+1-\sigma)^{2}$, then $E_{2}$ is a hyperbolic saddle for any choices of the parameters. And at $E_{3}$, we have $\phi_{3}>0$. Thus, the stability of the equilibrium $E_{3}$ depends on the sign of $\psi_{3}$.

Theorem 2.6 When $R_{0}=1$ and $c>\frac{1-\sigma}{a}$, then system (2.1) has a unique positive equilibrium $E_{4}\left(x_{4}, y_{4}\right)$, and the equilibrium $E_{4}$ is stable if $\psi_{4}<0$.

Proof In fact, when $R_{0}=1$ and $c>\frac{1-\sigma}{a}$, then $\phi_{4}>0$. Thus, the stability of $E_{4}$ is determined by the sign of $\psi_{4}$. 
(a)

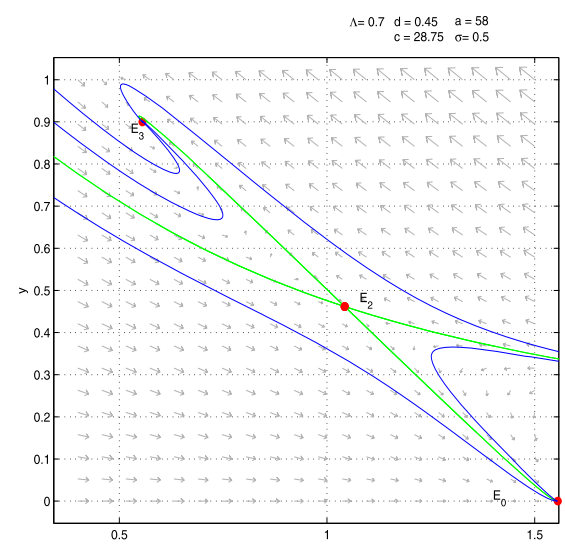

(b)

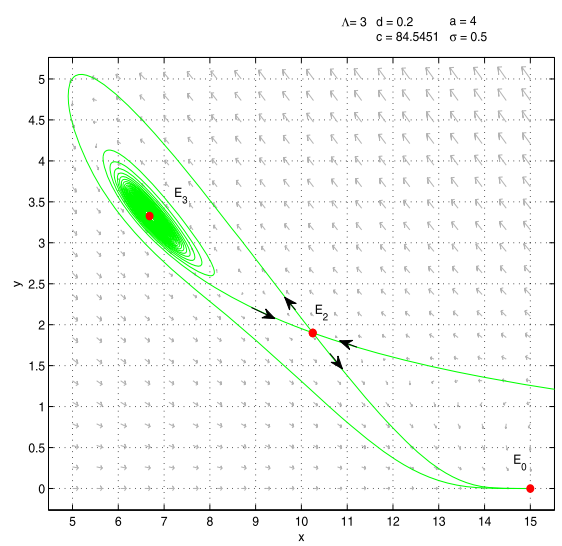

Figure 2 When $R^{*}<R_{0}<1, \Lambda>(1-\sigma) / a$. (a) $\psi_{3}<0$, equilibria $E_{0}$ and $E_{3}$ are locally stable and $E_{2}$ is unstable. (b) $\psi_{3}>0$, equilibrium $E_{0}$ is locally stable and $E_{2}$ and $E_{3}$ are unstable

Theorem 2.7 Assume $R_{0}>1$, then system (2.1) has a unique positive equilibrium $E_{5}$. Moreover,

(i) $E_{5}$ is stable if $\psi_{5}<0$;

(ii) $E_{5}$ is a weak focus or center if $\psi_{5}=0$;

(iii) $E_{5}$ is unstable if $\psi_{5}>0$.

Proof Obviously, when $R_{0}>1$, then $\phi_{5}>0$, and then $E_{5}$ is stable if $\psi_{5}<0$.

Lemma 2.8 From the expression of $\psi_{k}(k=1,3,5)$, we can see that $E_{k}(k=1,3,5)$ is always stable if $d \geq 1-\frac{1}{a c}$.

When $\psi_{i} \neq 0(i=3,5)$, the dynamics of system (2.1) can easily be seen in Fig. 2, Fig. 3 and Fig. 4, respectively. The dynamical behaviors of system $(2.1)$ when $\psi_{i}=0(i=3,5)$ will be discussed in detail in the next section.

Remark 2.9 In fact, Fig. 2(a) shows the occurrence of bi-stability, in which solution may converge to one of the two equilibria, depending on the initial conditions. And in practical cases, this interesting phenomenon implies that initial states determine whether the disease dies out or not.

Remark 2.10 From Fig. 2(a), we can see that there exist two separatrices. All solutions tend to the disease-free equilibrium $E_{0}$ except the two green lines tend to equilibrium $E_{2}$.

Theorem 2.11 Suppose that $R_{0}=1$ and $\Lambda>\frac{1-\sigma}{a}$, then system (2.1) has a unique positive equilibrium $E_{4}$. If $\psi_{4}>0$, then there exists at least one stable limit cycle in the interior of the first quadrant.

Proof Indeed, the Jacobian $J_{0}$ is diagonalizable with eigenvalues $\lambda_{1}=-d$ and $\lambda_{2}=0$ and respective eigenvectors $v_{1}=(1,0)$ and $v_{2}=\left(-\frac{1-\sigma}{d}, 1\right)$. By the transformation

$$
\left(\begin{array}{l}
u \\
v
\end{array}\right)=\left(\begin{array}{cc}
1 & -\frac{1-\sigma}{d} \\
0 & 1
\end{array}\right)\left(\begin{array}{l}
x \\
y
\end{array}\right),
$$


(a)

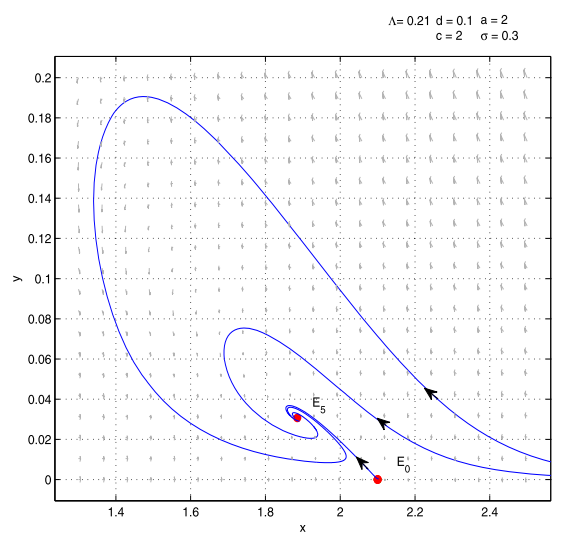

(b)

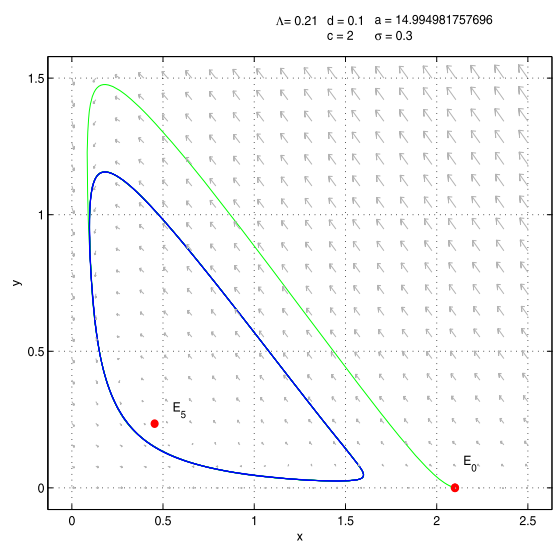

Figure 3 When $R_{0}>1$. (a) $\psi_{5}<0$, the disease-free equilibrium $E_{0}$ is unstable and the unique positive equilibrium $E_{5}$ is globally stable. (b) $\psi_{5}>0, E_{0}$ and $E_{5}$ are unstable and there exists a stable limit cycle

(a)

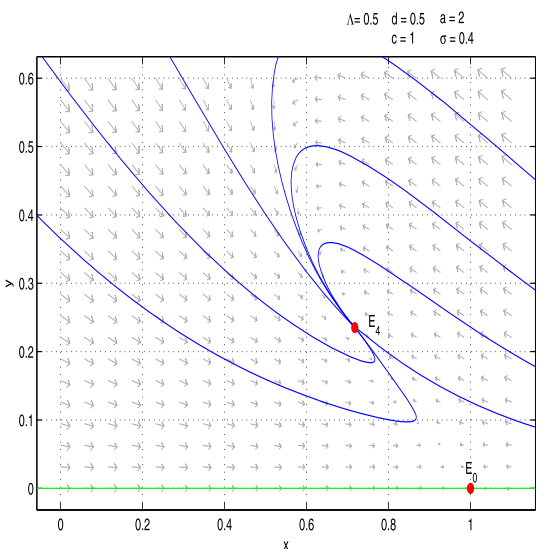

(b)

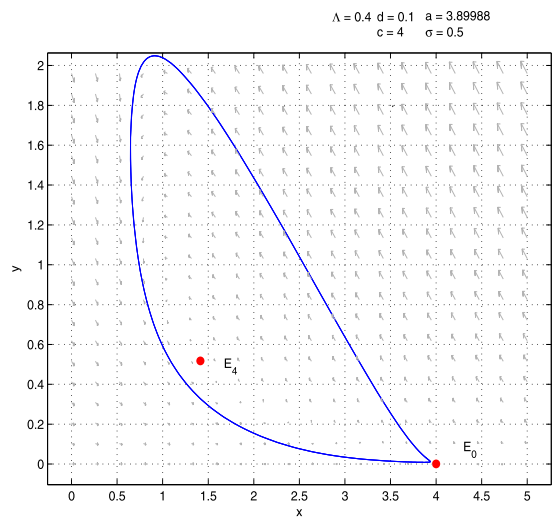

Figure 4 When $R_{0}=1$, and $\Lambda>(1-\sigma) / a$. (a) $\psi_{4}<0$, the disease-free equilibrium $E_{0}$ is unstable and the unique positive equilibrium $E_{4}$ is globally stable. (b) $\psi_{4}>0, E_{0}$ and $E_{4}$ are unstable and there exists a stable limit cycle

system (2.3) becomes

$$
\left\{\begin{array}{l}
\frac{d x}{d t}=-d x-\left(\frac{1}{c}+\frac{1-\sigma}{d}\right) x y-\left(\frac{1-\sigma}{c d}-2 a+\frac{2 a(1-\sigma)}{d}\right) y^{2}+\mathcal{O}\left(|(x, y)|^{3}\right) \\
\frac{d y}{d t}=\left(2 a-\frac{1-\sigma}{d c}\right) y^{2}+\frac{1}{c} x y+\mathcal{O}\left(|(x, y)|^{3}\right) .
\end{array}\right.
$$

The theorem of Chochitaichvili [15] shows directly that system (2.4) is topologically equivalent to the system

$$
\left\{\begin{array}{l}
\frac{d x}{d t}=-d x \\
\frac{d y}{d t}=\left(2 a-\frac{1-\sigma}{d c}\right) y^{2}+\mathcal{O}\left(|y|^{3}\right)
\end{array}\right.
$$


(a)

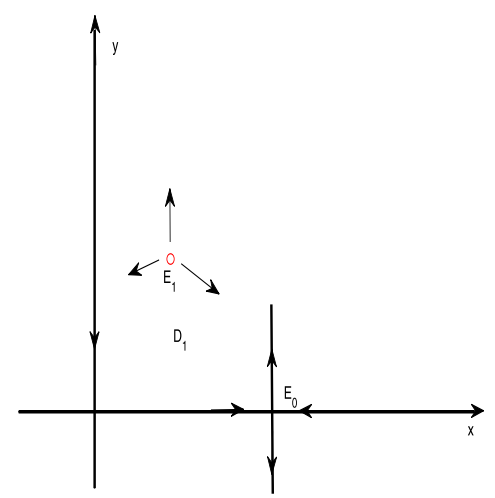

(b)

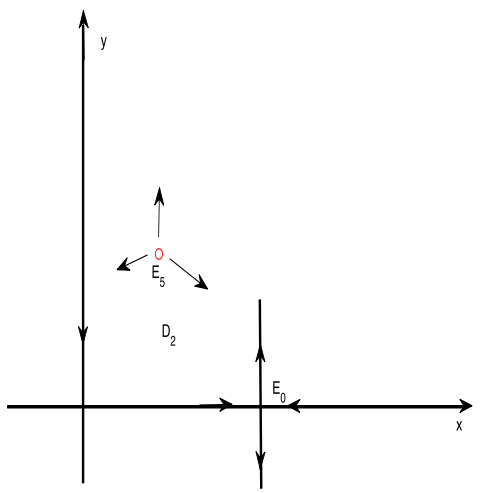

Figure 5 When $R_{0}=1$, and $\Lambda>(1-\sigma) / a$. (a) When $R_{0}=1, \Lambda>\frac{1-\sigma}{a}$ and $\psi_{4}>0$, there exists a stable limit cycle. (b) When $R_{0}>1$ and $\psi_{5}>0$, there exists a stable limit cycle

It is easy to see that $2 a-\frac{1-\sigma}{d c}>0$ according to the condition of $R_{0}=1$ and $\Lambda>\frac{1-\sigma}{a}$, and then we find that there exists a unique repelling equilibrium $E_{4}$ in the region $D_{1}$ shown in (a) of Fig. 5. Consequently, by the Poincaré-Bendixson theorem, at least one stable limit cycle appears in the interior of the first quadrant.

Similarly, when $\psi_{5}>0$, we have the following result.

Theorem 2.12 Suppose that $R_{0}>1$. If $\psi_{5}>0$, then there exists at least one stable limit cycle in the interior of the first quadrant.

Proof Indeed, the Jacobian $J_{5}$ has eigenvalues $\lambda_{1}=-d$ and $\lambda_{2}=R_{0}-1>0$, when $R_{0}>1$. Thus, we find that there exists a $E_{5}$, which is the unique repelling equilibrium in the region $D_{2}$ shown in (b) of Fig. 5. Consequently, by the Poincaré-Bendixson theorem, at least one stable limit cycle appears in the interior of the first quadrant.

Remark 2.13 From (b) of Fig. 3, we can see clearly that there exists a stable limit cycle enclosing the equilibrium $E_{5}$ even though $E_{0}$ is a saddle node. Similarly, from (b) of Fig. 4, we also find that there exists a stable limit cycle if $E_{4}$ is unstable.

\section{Bifurcations}

\subsection{Backward bifurcation}

Theorem 3.1 When $R_{0}=1$ and $\Lambda>\frac{1-\sigma}{a}$, model (2.1) exhibits a backward bifurcation at equilibrium $E_{0}$.

Remark 3.2 When $R_{0}=1$ and $\Lambda>\frac{1-\sigma}{a}$, system (2.1) exhibits a unique positive equilibrium $E_{4}$, which means that once $R_{0}$ crosses 1 , the disease can invade to a relatively high level. And this is one of the main characters of backward bifurcation [16].

Remark 3.3 Actually, backward bifurcation did not emerge with $a=0$, which is considered in [13]. This indicates that introducing the non-motonic incidence into model (1.1) makes the epidemic model more complex and exhibits richer dynamical behaviors. 


\subsection{Hopf bifurcation}

In this subsection, we will study the Hopf bifurcation of system (2.1) for (i) $R^{*}<R_{0}<1$ and $\Lambda>(1-\sigma) / a$; (ii) $R_{0}>1$. From the discussion in Sect. 2, it can be seen that Hopf bifurcation may occur at $E_{3}, E_{5}$. The expressions of the equilibria $E_{3}$ and $E_{5}$ are the same, not considering the values of every parameters. Based on Theorem 2.5, Theorem 2.6 and Theorem 2.7, we know that the stability of $E_{3}$ and that of $E_{5}$ are similar and when $\psi_{k}=0$, $E_{k}(k=3,5)$ is a weak focus or center. Thus, we show the existence of a Hopf bifurcation around $E_{k}(k=3,5)$.

Theorem 3.4 Suppose $E_{k}(k=3,5)$ exist, then model (1.2) undergoes a Hopf bifurcation at equilibrium $E_{k}$ if $\psi_{k}=0$. Moreover,

(a) if $\eta<0$, there is a family of stable periodic orbits of model (2.1) as $\psi_{k}$ decreases from 0 ;

(b) if $\eta=0$, there are at least two limit cycles in (2.1), where $\eta$ will be defined below;

(c) if $\eta>0$, there is a family of unstable periodic orbits of (2.1) as $\psi_{k}$ increases from 0.

Proof From the above discussions, we can see that $\operatorname{tr} J_{k}=0$ if and only if $\psi_{k}=0$, and $\operatorname{det} J_{k}>$ 0 when equilibrium $E_{k}$ exists. Therefore, the eigenvalues of $J_{k}$ are a pair of pure imaginary roots if $\psi_{k}=0$. From direct calculations we have

$$
\left.\frac{d\left(\operatorname{tr} J_{k}\right)}{d \psi_{k}}\right|_{\psi_{k}=0}=\frac{1}{\left(y_{k}^{2}+c\right)\left(y_{k}+1\right)} \neq 0 .
$$

By Theorem 3.4.2 in [17], $\Psi_{k}=0$ is the Hopf bifurcation point for (2.1).

Next, similar to [13], we introduce a new time variable $\tau$ by $d t=\left(y^{2}+c\right) d \tau$. By rewriting $\tau$ as $t$, we obtain the following equivalent system of (2.1):

$$
\left\{\begin{array}{l}
\frac{d x}{d t}=\Lambda\left(y^{2}+c\right)-x y(a y+1)-d x\left(y^{2}+c\right)+\sigma y\left(y^{2}+c\right), \\
\frac{d y}{d t}=x y(a y+1)-y\left(y^{2}+c\right) .
\end{array}\right.
$$

Let $X=x-x_{k}$ and $Y=y-y_{k}$, still use $(x, y)$ to express $(X, Y)$, then system (3.1) becomes

$$
\left\{\begin{array}{l}
\frac{d x}{d t}=b_{11} x+b_{12} y+c_{1} x y+c_{2} y^{2}+c_{3} x y^{2}+c_{4} y^{3} \\
\frac{d y}{d t}=b_{21} x+b_{22} y+c_{5} x y+c_{6} y^{2}+c_{7} x y^{2}+c_{8} y^{3}
\end{array}\right.
$$

where

$$
\begin{aligned}
& b_{11}=-d\left(\left(y_{k}\right)^{2}+c\right)-y_{k}\left(a y_{k}+1\right), \quad b_{21}=y_{k}\left(a y_{k}+1\right), \quad b_{22}=\left(a x_{k}-2 y_{k}\right) y_{k}, \\
& b_{12}=2 \Lambda y_{k}-2(a+d) x_{k} y_{k}-x_{k}+\sigma\left(3\left(y_{k}\right)^{2}+c\right), \quad c_{1}=-1-2(a+d) y_{k}, \\
& c_{2}=\Lambda+3 \sigma y_{k}-(a+d) x_{k}, \quad c_{3}=-(a+d), \quad c_{4}=\sigma, \quad c_{5}=2 a y_{k}+1, \\
& c_{6}=a x_{k}-3 y_{k}, \quad c_{7}=a, \quad c_{8}=-1 .
\end{aligned}
$$

Let $\hat{E}$ denote the origin of $x-y$ plane. Since $E_{k}$ satisfies Eq. (2.1), we obtain

$$
\operatorname{det} J(\hat{E})=b_{11} b_{22}-b_{12} b_{21}=\frac{y_{k} \psi_{k}}{y_{k}+b}>0
$$


and it is easy to verify that $b_{11}+b_{22}=0$ if and only if $\psi_{k}=0$. Let $\omega=(\operatorname{det} J(\hat{E}))^{\frac{1}{2}}, u=-x$ and $v=\frac{b_{11}}{\omega} x+\frac{b_{12}}{\omega} y$, then the normal form of system (3.1) reads

$$
\left\{\begin{array}{l}
\frac{d u}{d t}=-\omega v+f(u, v), \\
\frac{d v}{d t}=\omega u+g(u, v),
\end{array}\right.
$$

where

$$
\begin{aligned}
f(u, v)= & \left(\frac{b_{11} c_{1}}{b_{12}}-\frac{b_{11}^{2} c_{2}}{b_{12}^{2}}\right) u^{2}+\omega\left(\frac{c_{1}}{b_{12}}-\frac{2 b_{11} c_{2}}{b_{12}^{2}}\right) u v-\frac{c_{2} \omega^{2}}{b_{12}^{2}} v^{2}+b_{11}^{2}\left(\frac{c_{3}}{b_{12}^{2}}-\frac{b_{11} c_{4}}{b_{12}^{3}}\right) u^{3} \\
& +b_{11} \omega\left(\frac{2 c_{3}}{b_{12}^{2}}-\frac{3 b_{11} c_{4}}{b_{12}^{3}}\right) u^{2} v+\omega^{2}\left(\frac{c_{3}}{b_{12}^{2}}-\frac{3 b_{11} c_{4}}{b_{12}^{3}}\right) u v^{2}-\frac{c_{4} \omega^{3}}{b_{12}^{3}} v^{3}, \\
g(u, v)= & \frac{1}{\omega}\left(\frac{b_{11}^{3} c_{2}}{b_{12}^{2}}-\frac{b_{11}^{2} c_{1}}{b_{12}}-c_{5} b_{11}+\frac{b_{11}^{2} c_{6}}{b_{12}}\right) u^{2}+\left(\frac{2 b_{11}^{2} c_{2}}{b_{12}^{2}}-\frac{b_{11} c_{1}}{b_{12}}+\frac{2 b_{11} c_{6}}{b_{12}}-c_{5}\right) u v \\
& +\omega\left(\frac{b_{11} c_{2}}{b_{12}^{2}}+\frac{c_{6}}{b_{12}}\right) v^{2}+\frac{b_{11}^{2}}{b_{12} \omega}\left(\frac{b_{11}^{2} c_{4}}{b_{12}^{2}}-\frac{b_{11} c_{3}}{b_{12}}-c_{7}+\frac{b_{11} c_{8}}{b_{12}}\right) u^{3} \\
& +\left(\frac{3 b_{11}^{3} c_{4}}{b_{12}^{3}}-\frac{2 b_{11}^{2} c_{3}}{b_{12}^{2}}-\frac{b_{11} c_{7}}{b_{12}}+\frac{3 b_{11}^{2} c_{8}}{b_{12}^{2}}\right) u^{2} v \\
& +\omega\left(\frac{3 b_{11}^{2} c_{4}}{b_{12}^{3}}-\frac{b_{11} c_{3}}{b_{12}^{2}}-\frac{c_{7}}{b_{12}}+\frac{3 b_{11} c_{8}}{b_{12}^{2}}\right) u v^{2}+\omega^{2}\left(\frac{b_{11} c_{4}}{b_{12}^{3}}+\frac{c_{8}}{b_{12}^{2}}\right) v^{3} .
\end{aligned}
$$

Set

$$
\begin{aligned}
\Gamma= & \frac{1}{16}\left[f_{u u u}+f_{u v v}+g_{u u v}+g_{v v v}\right] \\
& +\frac{1}{16 \omega}\left[f_{u v}\left(f_{u u}+f_{v v}\right)-g_{u v}\left(g_{u u}+g_{v v}\right)-f_{u u} g_{u u}+f_{v v} g_{v v}\right]
\end{aligned}
$$

where $f_{u v}$ denotes $\frac{\partial^{2} f}{\partial u \partial v}(0,0)$, etc. Then by computing we obtain

$$
\Gamma=\frac{\eta}{8 b_{12}^{2} \omega^{2}}
$$

where

$$
\begin{aligned}
\eta= & {\left[c_{3}+3 c_{8}-\frac{c_{2}\left(c_{1}+2 c_{6}\right)}{b_{12}}\right] \omega^{4}+\left[\left(c_{3}+3 c_{8}-\frac{2 c_{2}\left(c_{1}+2 c_{6}\right)}{b_{12}}\right) b_{11}^{2}\right.} \\
& \left.+b_{11}\left(c_{1}^{2}-2 b_{12} c_{7}+c_{2} c_{5}+c_{1} c_{6}-2 c_{6}^{2}\right)+b_{12} c_{5} c_{6}\right] \omega^{2} \\
& +\left[c_{5}-\frac{b_{11}\left(c_{1}+2 c_{6}\right)}{b_{12}}\right]\left[b_{11}^{2}\left(b_{12} c_{6}-c_{1}\right)+b_{11}\left(b_{11}^{2} c_{2}-b_{12}^{2} c_{5}\right)\right] .
\end{aligned}
$$

By Theorem 3.4.2 and Theorem 3.4.11 in [17], the rest of the claims in Theorem 3.4 are proven.

Remark 3.5 What we need to note here is that the expression $b_{12}$ in Theorem 3.4 is nonzero. Otherwise, we have $\operatorname{det} J_{k}=b_{11} b_{22}<0$, since $b_{11}+b_{22}=0$, which is a contradiction. 
Next, we present examples to show that equilibrium $E_{k}$ can be a stable weak focus of multiplicity two, and under a small perturbation, system (2.1) undergoes a degenerate Hopf bifurcation and produces two limit cycles.

Firstly, fix $y_{k}=1 / 2$ and solve for $a=-2+\frac{6}{(2-2 \Lambda-\sigma)}$. Also, fix $x_{k}=1$, based on $a=$ $-2+\frac{6}{(2-2 \Lambda-\sigma)}$, we can get $d=\frac{1}{2(2 \Lambda+1-\sigma)}$. Then $\Psi_{k}=0$ if and only if $c=\frac{-10-2 l-\sigma}{4(2 \Lambda+\sigma-2)}$. Secondly, substituting these expressions into $\eta$ and through complicated computation, we obtain

$$
\begin{aligned}
\eta \triangleq & L_{1} \\
= & \frac{81(1+2 \Lambda+\bar{\sigma})^{2}}{64(1-2 \Lambda+\bar{\sigma})^{6}}\left(32 \Lambda^{5}-80 \Lambda^{4}(-1+\bar{\sigma})+8 \Lambda^{3}(-39+2 \bar{\sigma}(-13+5 \bar{\sigma}))\right. \\
& +4 \Lambda^{2}(-103+\bar{\sigma}(99+2(24-5 \bar{\sigma}) \bar{\sigma}))+\bar{\sigma}(140-\bar{\sigma}(139+\bar{\sigma}(-21 \\
& +(-11+\bar{\sigma}) \bar{\sigma})))+2 \Lambda(-80+\bar{\sigma}(242+\bar{\sigma}(-81+\bar{\sigma}(-38+5 \bar{\sigma}))))),
\end{aligned}
$$

with $\bar{\sigma}=1-\sigma>0$, which is the first Liapunov number of the equilibrium $(0,0)$ of (3.2). Then we fix $\bar{\sigma}=4 / 5$ and solve the equation $\eta=0$, then we get only one suitable value 0.5859 for $\Lambda$. That is to say, if $(\sigma, \Lambda, d, a, c)=(1 / 5,5859,0.2,8,4.741)$, then $L_{1}=0$. Furthermore, it can be seen that $E_{k}=E_{3}$ under this group of parameters.

In the following, we further compute the second Liapunov number of the equilibrium $(0,0)$ of system $(3.2)$ by the successor function method. It is convenient to introduce polar coordinates $(r, \theta)$ and rewrite system (3.2) in polar coordinates by $x=r \cos \theta, y=r \sin \theta$. It is clear that in a small neighborhood of the origin, the successor function $D\left(c_{0}\right)$ of system (3.2) can be expressed by

$$
D\left(c_{0}\right)=r\left(2 \pi, c_{0}\right)-r\left(0, c_{0}\right)
$$

where $r\left(\theta, c_{0}\right)$ is the solution of the following Cauchy problem:

$$
\begin{aligned}
& \frac{d r}{d \theta}=R_{2}(\theta) r^{2}+R_{3}(\theta) r^{3}+R_{4}(\theta) r^{4}+R_{5}(\theta) r^{5}+\cdots, \\
& r(0)=c_{0}, \quad 0<\left|c_{0}\right| \ll 1,
\end{aligned}
$$

where $R_{i}(\theta)(i=1,2,3, \ldots)$ is a polynomial of $(\sin \theta, \cos \theta)$, whose coefficients can be expressed by the coefficients of system (3.2). We omit them here, since the expressions are too long.

With the aid of Mathematica, we get

$$
L_{2} \doteq-0.334275
$$

and

$$
\left|\begin{array}{ll}
\frac{\partial \operatorname{tr} J_{3}}{\partial \Lambda} & \frac{\partial \operatorname{tr} J_{3}}{\partial c} \\
\frac{\partial L_{1}}{\partial \Lambda} & \frac{\partial L_{1}}{\partial c}
\end{array}\right|=-42,982.27165,
$$

when $(\sigma, \Lambda, d, a, c)=(1 / 5,5859,0.2,8,4.741)$. Therefore, the interior equilibrium $E_{3}$ is a stable weak focus of multiplicity two if $(\sigma, \Lambda, d, a, c)=(1 / 5,5859,0.2,8,4.741)$. The phase portraits of system (2.1) under this group of parameters are shown in Fig. 6. 
(a)

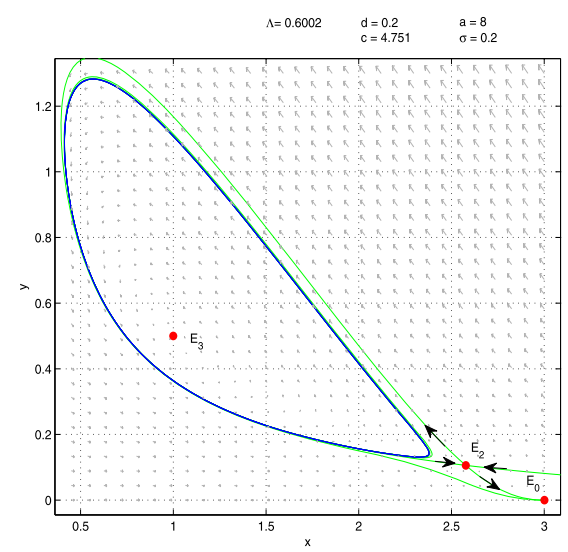

(b)

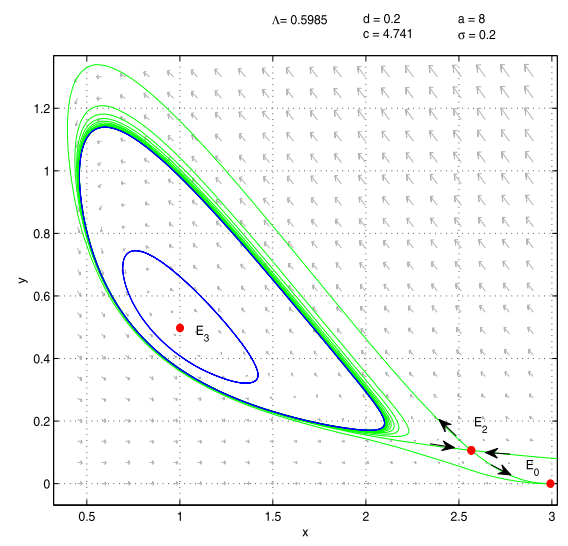

Figure 6 (a) One limit cycle enclosing an unstable hyperbolic focus $E_{3}$. (b) Two limit cycles enclosing an unstable hyperbolic focus $E_{3}$, and the small one is stable, the large one is unstable

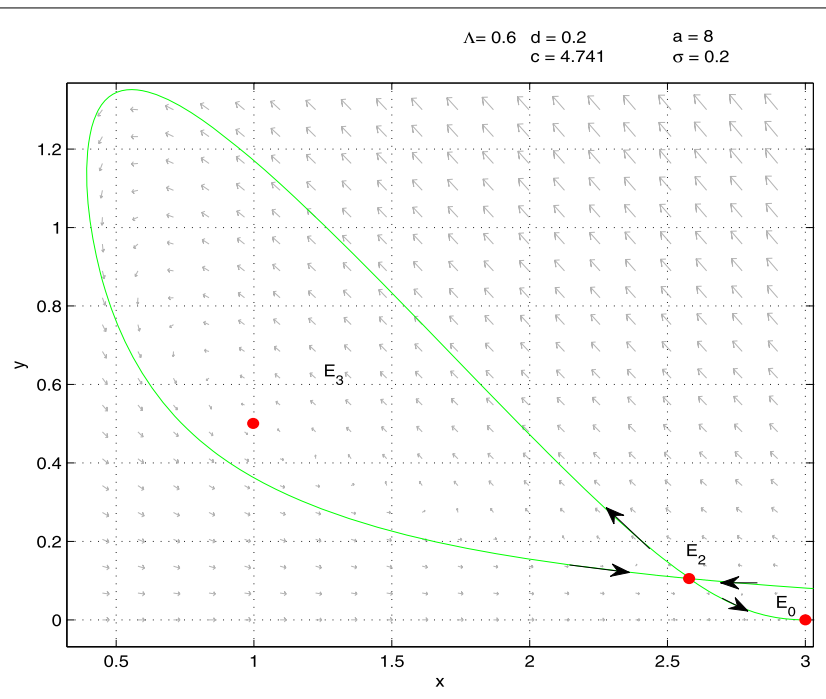

Figure 7 Equilibrium $E_{3}$ is stable and there exists an unstable homoclinic loop

Besides, we give the numerical simulation graphs for one limit cycle and two limit cycles under small perturbations of some parameters. From Fig. 6(a), we can see that there exists only one limit cycle around the endemic $E_{3}$, and Fig. 6(b) shows us that a new limit cycle emerges with small perturbations of the parameters $\Lambda$ and $c$. It is worth emphasizing that if we change the values of the parameters $\Lambda$ and $c$, an unstable homoclinic loop arise, which is shown in Fig. 7.

Around equilibrium $E_{5}$, we obtain the same result from Fig. 8. Under the condition that parameter $a, d, \sigma$ take value 5.6923, $0.11,0.2$ and change value of $\Lambda$ and $c$ from 0.45102 , 3.6062 to $0.51,3.5972$, respectively, which is a minor change, then the number of limit cycles will add one. Thus, the interior equilibrium $E_{5}$ is a stable weak focus of multiplicity two if $(\sigma, \Lambda, d, a, c)=(0.2,0.51,0.11,5.6923,3.5962)$. 
(a)

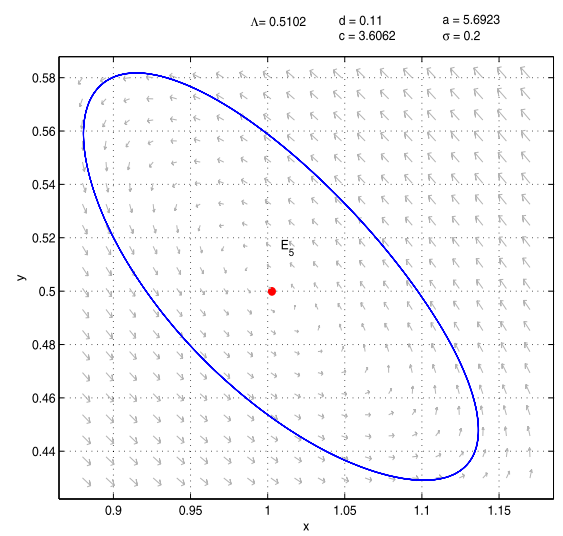

(b)

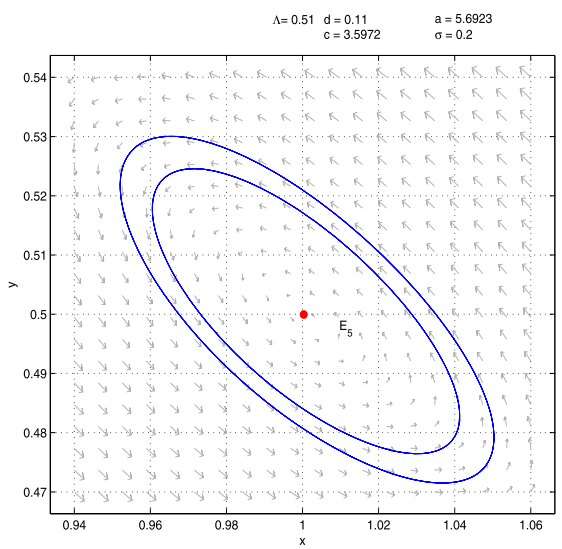

Figure 8 (a) One limit cycle enclosing an unstable hyperbolic focus $E_{5}$. (b) Two limit cycles enclosing an unstable hyperbolic focus $E_{5}$, and the small one is stable, the large one is unstable

Remark 3.6 As a matter of fact, the reproduction number is equal to zero in [13, 18], which simplifies the condition that a Hopf bifurcation occur. In our model, we also comprehensively discuss the existence of a Hopf bifurcation when $R_{0}<1, R_{0}=1$ and $R_{0}>1$. Besides, the authors in [13] did not show the appearance of a homoclinic loop, which is an interesting bifurcation phenomenon given in Fig. 7.

\subsection{Bogdanov-Takens bifurcation}

In this subsection, we investigate the Bogdanov-Takens bifurcation in system (2.1). Lemma 3.7 is from Perko [19], and Lemma 3.8 is Proposition 5.3 in Lamontage et al. [20].

\section{Lemma 3.7 The system}

$$
\left\{\begin{array}{l}
\frac{d x}{d t}=y+A x^{2}+B x y+C y^{2}+\mathcal{O}\left(|(X, Y)|^{3}\right), \\
\frac{d y}{d t}=D x^{2}+E x y+F y^{2}+\mathcal{O}\left(|(X, Y)|^{3}\right)
\end{array}\right.
$$

is equivalent to the system

$$
\left\{\begin{array}{l}
\frac{d x}{d t}=y, \\
\frac{d y}{d t}=D x^{2}+(E+2 A) x y+\mathcal{O}\left(|(X, Y)|^{3}\right),
\end{array}\right.
$$

in some small neighborhood of $(0,0)$ after changes of coordinates.

Lemma 3.8 The system

$$
\left\{\begin{array}{l}
\frac{d x}{d t}=y, \\
\frac{d y}{d t}=x^{2}+a_{30} x^{3}+a_{40} x^{4}+y\left(a_{21} x^{2}+a_{31} x^{3}\right)+y^{2}\left(a_{12} x+a_{22} x^{2}\right)+\mathcal{O}\left(|(x, y)|^{4}\right),
\end{array}\right.
$$


is equivalent to the system

$$
\left\{\begin{array}{l}
\frac{d x}{d t}=y \\
\frac{d y}{d t}=x^{2}+\left(a_{31}-a_{30} a_{21}\right) x y+\mathcal{O}\left(|(X, Y)|^{3}\right),
\end{array}\right.
$$

in some small neighborhood of $(0,0)$ after changes of coordinates.

From Theorem 2.2, we can see that there exists a unique positive equilibrium $E_{1}\left(x_{1}, y_{1}\right)$ when $R_{0}=R^{*}$, where

$$
x_{1}=\frac{y_{1}^{2}+c}{a y_{1}+1}, \quad y_{1}=\frac{a \Lambda-(1-\sigma)}{2(d+a(1-\sigma))} .
$$

From the proving process of Theorem 2.5 , it is easily to see that $\operatorname{det} J_{1}=0$. And Theorem 2.4 suggests that the characteristic matrix $J_{1}$ possesses a zero eigenvalue with multiplicity 2 when $\psi_{1}=0$, which shows that system (2.1) may admit a Bogdanov-Takens bifurcation. Thus, we can prove the following theorem.

Define two functions:

$$
\begin{aligned}
f(y)= & (1+a+d) y^{4}+(1-a c(3+2 a+2 d)) y^{3}-3 c(1+a) y^{2} \\
& -c(1+a c(-1+2 d)) y-c^{2} d \\
g(y)= & c^{2} d+2 c(1+6 a-2 c+c d) y+6 c(2+a) y^{2} \\
& +2\left(2 a^{2} c+a c d-1\right) y^{3}-(4+2 a+d) y^{4}
\end{aligned}
$$

Theorem 3.9 Suppose that $R_{0}=R^{*}$ and $\psi_{1}=0$, then the only interior equilibrium $E_{1}$ of system (2.1) is a cusp. Moreover,

(a) if $f\left(y_{1}\right) g\left(y_{1}\right) \neq 0$, then $E_{1}$ is a cusp of codimension 2 ;

(b) iff $\left(y_{1}\right) g\left(y_{1}\right)=0$, then $E_{1}$ is a cusp of codimension greater than or equal to 3 .

Proof Changing the variables as $X=x-x_{1}, Y=y-y_{1}$, system (2.1) becomes

$$
\left\{\begin{array}{l}
\frac{d X}{d t}=b_{11} X+b_{12} Y+c_{1} X Y+c_{3} Y^{2}+\mathcal{O}\left(|(X, Y)|^{3}\right) \\
\frac{d Y}{d t}=b_{21} X+b_{22} Y-c_{1} X Y+c_{2} Y^{2}+\mathcal{O}\left(|(X, Y)|^{3}\right)
\end{array}\right.
$$

where

$$
\begin{aligned}
& b_{11}=-d-\frac{y_{1}\left(a y_{1}+1\right)}{y_{4}^{2}+c}, \quad b_{12}=\sigma-\frac{-y_{1}^{2}+2 a c y_{1}+c}{\left(y_{1}^{2}+c\right)^{2}} x_{1}, \quad b_{21}=\frac{y_{1}\left(a y_{1}+1\right)}{y_{4}^{2}+c}, \\
& b_{22}=-1+\frac{-y_{1}^{2}+2 a c y_{1}+c}{\left(y_{1}^{2}+c\right)^{2}} x_{1}, \quad c_{1}=-\frac{-y_{1}^{2}+2 a c y_{1}+c}{\left(y_{1}^{2}+c\right)^{2}}, \\
& c_{2}=\frac{2\left(y_{1}^{3}-3 a c y_{1}^{2}-3 c y_{1}+a c^{2}\right) x_{1}}{\left(y_{1}^{2}+c\right)^{3}}, \quad c_{3}=-\frac{x_{1}\left(a c^{2}-3 c y_{1}-3 a c y_{1}^{2}+y_{1}^{3}\right)}{\left(c+y_{1}^{2}\right)^{3}} .
\end{aligned}
$$

Make the non-singular linear transformation

$$
\left(\begin{array}{l}
X \\
Y
\end{array}\right)=\left(\begin{array}{cc}
\frac{b_{11}}{b_{21}} & \frac{1}{b_{21}} \\
1 & 0
\end{array}\right)\left(\begin{array}{l}
x \\
y
\end{array}\right)
$$


Then system (3.3) is transformed into

$$
\left\{\begin{array}{l}
\frac{d x}{d t}=y+b_{1} x^{2}+b_{2} x y \\
\frac{d y}{d t}=b_{3} x^{2}+b_{4} x y+Q_{2}(x, y)
\end{array}\right.
$$

where $Q_{2}(x, y)$ is a smooth function in $(x, y)$ at least of the third order and

$$
b_{1}=c_{2}-\frac{c_{1} b_{11}}{b_{21}}, \quad b_{2}=-\frac{c_{1}}{b_{21}}, \quad b_{3}=b_{21} c_{3}+b_{11}\left(c_{1}-c_{2}\right)+\frac{b_{11}^{2} c_{1}}{b_{21}}, \quad b_{4}=\frac{-d c_{1}}{b_{21}} \text {. }
$$

By Lemma 3.7, we obtain a topologically equivalent system of system (3.4)

$$
\left\{\begin{array}{l}
\frac{d u}{d t}=v \\
\frac{d v}{d t}=b_{3} u^{2}+\left(b_{4}+2 b_{1}\right) u v+Q_{3}(u, v)
\end{array}\right.
$$

where

$$
b_{3}=\frac{d f\left(y_{1}\right)}{\left(c+y_{1}^{2}\right)^{3}}, \quad b_{4}+2 b_{1}=-\frac{g\left(y_{1}\right)}{y_{1}\left(a y_{1}+1\right)\left(y_{1}^{2}+c\right)^{2}} .
$$

Therefore, $E_{1}$ is a cusp of codimension 2 if $f\left(y_{1}\right) g\left(y_{1}\right) \neq 0$, by the results in Perko [19], or else, $E_{1}$ is a cusp of codimension at least 3 .

Remark 3.10 In fact, Theorem 3.9(b) includes the following three cases:

(1) If $f\left(y_{1}\right) \neq 0$ and $g\left(y_{1}\right)=0, E_{1}$ is a cusp point;

(2) If $f\left(y_{1}\right)=0$ and $g\left(y_{1}\right) \neq 0, E_{1}$ is nilpotent focus/elliptic point;

(3) If $f\left(y_{1}\right)=g\left(y_{1}\right)=0, E_{1}$ is a nilpotent focus.

Unfortunately, due to the complexity of $f\left(y_{1}\right)$ and $g\left(y_{1}\right)$, we cannot determine which of these three situations occurs theoretically. But we will show for some parameter values that $f\left(y_{1}\right) \neq 0$ and $g\left(y_{1}\right)=0$, i.e. $E_{1}$ is a cusp point.

In the following, we will give an example to show that Theorem 3.9(b) occurs.

In the first place, fix $y_{1}=1 / 5$, then we can solve for $\sigma=1-\frac{-2 d+5 a \Lambda}{5+2 a}$. By assumptions $\psi_{1}=0$ and $R_{0}=R^{*}$, solve for parameters $d$ and $\Lambda$,

$$
d=\frac{(5+a)^{2} \Lambda}{-1+25 c+10 a c}, \quad \Lambda=\frac{(35+a(11+a-25 c))(1-5(5+2 a) c)}{(5+a)^{3}(1+25 c)} .
$$

Then

$$
f\left(y_{1}\right)=-\frac{(1+25 c)\left(1+a^{2} c\right)}{125(5+a)}<0
$$

for any $a, c>0$. We solve $g\left(y_{1}\right)=0$ for the parameter $c$ and denote the corresponding solution with respect to $c$ by $c_{ \pm}^{g}$, where

$$
c_{ \pm}^{g}=\frac{(375+a(1630+a(323+2 a)) \pm \sqrt{(5+a)(28,125+a(236,775+a(531,415+a(104,449+4 a(318+a)))))})}{50 a(15+2 a)} .
$$


Actually, if $c=c_{-}^{g}$, we have $d=d(a)<0$ for any $a>0$. Thus, $g\left(y_{1}\right)=0$ if and only if $c=c_{+}^{g} \triangleq$ $c^{g}(a)$. In addition, with the help of Mathematica, for any $0<a<1.86433$ or $a>123.449$, one can have all other parameters positive and satisfying $d+\sigma<1$.

For example, take $a=1$, then we can get

$$
\begin{aligned}
(a, d, c, \Lambda, \sigma) & \doteq(1,0.108676,5.478144,0.575787,0.619774) \\
& \triangleq\left(a_{0}, d_{0}, c_{0}, \Lambda_{0}, \sigma_{0}\right)
\end{aligned}
$$

which satisfies $c=c^{g}(a)$, i.e. Theorem 3.9(b) is in order.

Theorem 3.11 When $(a, d, c, \Lambda, \sigma)=\left(a_{0}, d_{0}, c_{0}, \Lambda_{0}, \sigma_{0}\right)$, then $E_{1}$ is a Bogdanov-Takens point of codimension 3 , and system (2.1) localized at $E_{1}$ is topologically equivalent to

$$
\left\{\begin{array}{l}
\frac{d x}{d t}=y \\
\frac{d y}{d t}=x^{2}+G x^{3} y+\mathcal{O}\left(|(x, y)|^{3}\right)
\end{array}\right.
$$

where $G<0$.

Proof First of all, applying a linear transformation $T_{1}:(x, y) \rightarrow(u, v)$, defined by $u=x-x_{1}$, $v=y-y_{1}$, we can reduce system (2.1) further to the form

$$
\left\{\begin{array}{l}
\frac{d u}{d t}=p_{10} u+p_{01} v+\sum_{2 \leq i+j \leq 4} p_{i j} u^{i} v^{j}+\mathcal{O}\left(|(u, v)|^{5}\right) \\
\frac{d v}{d t}=q_{10} u+q_{01} v+\sum_{2 \leq i+j \leq 4} q_{i j} u^{i} v^{j}+\mathcal{O}\left(|(u, v)|^{5}\right)
\end{array}\right.
$$

where

$$
\begin{aligned}
& p_{10}=-d-\frac{y_{1}\left(a y_{1}+1\right)}{y_{4}^{2}+c}, \quad p_{01}=\sigma-\frac{-y_{1}^{2}+2 a c y_{1}+c}{\left(y_{1}^{2}+c\right)^{2}} x_{1}, \quad p_{11}=-\frac{-y_{1}^{2}+2 a c y_{1}+c}{\left(y_{1}^{2}+c\right)^{2}} \\
& p_{02}=-\frac{x_{1}\left(a c^{2}-3 a c y_{1}^{2}-3 c y_{1}+y_{1}^{3}\right)}{\left(c+y_{1}^{2}\right)^{3}}, \quad p_{12}=\frac{-a c^{2}+3 a c y_{1}^{2}+3 c y_{1}-y_{1}^{3}}{\left(c+y_{1}^{2}\right)^{3}}, \\
& p_{03}=\frac{x_{1}\left(4 a c^{2} y_{1}-4 a c y_{1}^{3}+c^{2}-6 c y_{1}^{2}+y_{1}^{4}\right)}{\left(c+y_{1}^{2}\right)^{4}} \\
& p_{13}=\frac{v^{3}\left(4 a c^{2} y_{1}-4 a c y_{1}^{3}+c^{2}-6 c y_{1}^{2}+y_{1}^{4}\right)}{\left(c+y_{1}^{2}\right)^{4}} \\
& p_{04}=\frac{x_{1}\left(a c^{3}-10 a c^{2} y_{1}^{2}+5 a c y_{1}^{4}-5 c^{2} y_{1}+10 c y_{1}^{3}-y_{1}^{5}\right)}{\left(c+y_{1}^{2}\right)^{5}} \text {, } \\
& q_{10}=\frac{y_{1}\left(a y_{1}+1\right)}{y_{4}^{2}+c}, \quad q_{01}=-1+\frac{-y_{1}^{2}+2 a c y_{1}+c}{\left(y_{1}^{2}+c\right)^{2}} x_{1}, \quad q_{11}=\frac{2 a c y_{1}+c-y_{1}^{2}}{\left(c+y_{1}^{2}\right)^{2}}, \\
& q_{02}=\frac{x_{1}\left(a c^{2}-3 a c y_{1}^{2}-3 c y_{1}+y_{1}^{3}\right)}{\left(c+y_{1}^{2}\right)^{3}}, \quad q_{12}=\frac{\left(a c^{2}-3 a c y_{1}^{2}-3 c y_{1}+y_{1}^{3}\right)}{\left(c+y_{1}^{2}\right)^{3}}, \\
& q_{03}=\frac{x_{1}\left(4 a c^{2} y_{1}-4 a c y_{1}^{3}+c^{2}-6 c y_{1}^{2}+y_{1}^{4}\right)}{\left(c+y_{1}^{2}\right)^{4}}, \\
& q_{13}=\frac{\left(-4 a c^{2} y_{1}+4 a c y_{1}^{3}-c^{2}+6 c y_{1}^{2}-y_{1}^{4}\right)}{\left(c+y_{1}^{2}\right)^{4}} \text {, }
\end{aligned}
$$




$$
\begin{aligned}
& q_{04}=\frac{x_{1}\left(a c^{3}-10 a c^{2} y_{1}^{2}+5 a c y_{1}^{4}-5 c^{2} y_{1}+10 c y_{1}^{3}-y_{1}^{5}\right)}{\left(c+y_{1}^{2}\right)^{5}} \\
& p_{20}=p_{21}=p_{22}=p_{30}=p_{31}=p_{40}=q_{20}=q_{21}=q_{22}=q_{30}=q_{31}=q_{40}=0 .
\end{aligned}
$$

Another transformation $T_{2}:(u, v) \rightarrow(x, y)$, defined by $x=v, y=q_{10} u-p_{10} v$, reduces system (3.5) to

$$
\left\{\begin{array}{l}
\frac{d x}{d t}=y+\sum_{2 \leq i+j \leq 4} a_{i j} x^{i} y^{j}+\mathcal{O}\left(|(x, y)|^{5}\right) \\
\frac{d y}{d t}=\sum_{2 \leq i+j \leq 4} b_{i j} x^{i} y^{j}+\mathcal{O}\left(|(x, y)|^{5}\right)
\end{array}\right.
$$

where

$$
\begin{aligned}
& a_{11}=\frac{q_{11}}{q_{10}}, \quad a_{20}=q_{02}+\frac{p_{10} q_{11}}{q_{10}}, \quad a_{21}=\frac{q_{12}}{q_{10}}, \quad a_{30}=\frac{q_{03} q_{10}+p_{10} q_{12}}{q_{10}} \\
& a_{40}=q_{04}, \quad b_{11}=p_{11}-\frac{p_{10} q_{11}}{q_{10}}, \quad b_{20}=p_{10} p_{11}-p_{10} q_{02}+p_{02} q_{10}-\frac{p_{10}^{2} q_{11}}{q_{10}} \\
& b_{21}=p_{12}-\frac{p_{10} q_{12}}{q_{10}}, \quad b_{30}=p_{10}\left(p_{12}-q_{03}\right)+p_{03} q_{10}-\frac{p_{10}^{2} q_{12}}{q_{10}}, \\
& b_{40}=-p_{10} q_{04}+p_{04} q_{10},
\end{aligned}
$$

and the other coefficients are equal to zero.

Then using the near-identity transformation $T_{3}:(x, y) \rightarrow(u, v)$, defined by $u=x+\frac{1}{2}\left(a_{11}+\right.$ $\left.b_{02}\right) x^{2}-a_{02} x y, v=y+a_{20} x^{2}-b_{20} x y$, and parameters $\left(a_{0}, d_{0}, c_{0}, \Lambda_{0}, \sigma_{0}\right)$ make $b_{11}+2 a_{20}=0$, so we obtain

$$
\left\{\begin{array}{l}
\frac{d u}{d t}=v+\sum_{3 \leq i+j \leq 4} d_{i j} u^{i} v^{j}+\mathcal{O}\left(|(u, v)|^{5}\right), \\
\frac{d v}{d t}=b_{20} u^{2}+\sum_{3 \leq i+j \leq 4} e_{i j} x^{i} y^{j}+\mathcal{O}\left(|(u, v)|^{5}\right),
\end{array}\right.
$$

where

$$
\begin{array}{rl}
d_{30}= & a_{30}+a_{20}\left(a_{11}+b_{02}\right)-a_{02} b_{20}, \quad d_{21}=-a_{02}\left(a_{20}-b_{11}\right)+a_{21}+a_{11}\left(a_{11}+b_{02}\right) \\
d_{12}= & 2 a_{12}, \quad d_{13}=a_{13}+a_{03}\left(a_{11}+4 b_{02}\right)-a_{02}\left(3 a_{02} b_{02}+b 03\right), \quad d_{03}=-a_{02}^{2}+a_{03} \\
d_{40}= & a_{40}-\left(a_{11}+b_{02}\right)\left(a_{11} a_{20}+\frac{1}{2} a_{30}-\frac{3}{2} a_{02} b_{20}\right)-\frac{3}{2} a_{20}\left(a_{11}+b_{02}\right)^{2} \\
& -a_{20} a_{21}-a_{02} b_{30} \\
d_{31}= & a_{31}-a_{11}^{3}+a_{02}\left(2 a_{30}+3 a_{20} b_{02}+a_{11}\left(4 a_{20}+b_{11}\right)-b_{21}-3 a_{02} b_{20}\right) \\
& +b_{02}\left(a_{21}-a_{11}^{2}\right)-2 a_{12} a_{20}, \\
d_{22}= & a_{22}-3 a_{03} a_{20}+\frac{1}{2} a_{12}\left(a_{11}+5 b_{02}\right)+a_{02}^{2}\left(a_{20}-2 b_{11}\right) \\
& +a_{02}\left(a_{21}+2 a_{11}\left(a_{11}+b_{02}\right)-b_{12}\right) \\
d_{04}= & -a_{02} a_{03}+a_{04}, \\
e_{30}=b_{30}-a_{20} b_{11}-\left(a_{11}+2 b_{02}\right) b_{20} & 1 \\
e_{21}= & a_{11} a_{20}-\frac{1}{2}\left(a_{11}+b_{02}\right) b_{11}+2 a_{02} b_{20}+b_{21}
\end{array}
$$




$$
\begin{aligned}
e_{12}= & -b_{02}\left(a_{11}+b_{02}\right)+a_{02}\left(4 a_{20}+b_{11}\right)+b_{12}, \\
e_{03}= & a_{02} a_{20}+b_{03}, \quad e_{04}=b_{04}+a_{03} a_{20}, \\
e_{40}= & a_{20}\left(2\left(a_{30}-a_{11} a_{20}-a_{02} b_{20}\right)-a_{20} b_{02}-b_{21}\right) \\
& +\frac{3}{2}\left(a_{11}+b_{02}\right)\left(a_{20} b_{11}+b_{02} b_{20}-b_{30}\right)+\frac{5}{4}\left(a_{11}+b_{02}\right)^{2} b_{20}-b_{02} b_{30}+b_{40}, \\
e_{31}= & a_{20}\left(2 a_{21}-2 b_{12}+3 b_{02}^{2}\right)-b_{02}\left(a_{30}+b_{21}\right)+\frac{b_{02}^{2} b_{11}}{2}+\frac{1}{2} a_{11}^{2}\left(-2 a_{20}+b_{11}\right) \\
& +a_{11}\left(b_{02}\left(3 a_{20}+b_{11}\right)-4 a_{02} b_{20}-b_{21}\right) \\
& -a_{02}\left(a_{20}\left(6 a_{20}+4 b_{11}\right)+5 b_{02} b_{20}-3 b_{30}\right)+b_{31}, \\
& 1 \\
e_{22}= & \left(a_{20}\left(4 a_{12}-6 b_{03}\right)+\left(a_{11}^{2}-2 a_{21}+14 a_{02} a_{20}+b_{12}-3 b_{02}^{2}\right) b_{02}\right. \\
& \left.+\left(6 a_{02} b_{20}+4 b_{21}-b_{02} b_{11}\right) a_{02}+2 b_{22}-a_{11}\left(2 b_{02}^{2}+3 a_{02} b_{11}+b_{12}\right)\right), \\
e_{13}= & 2 a_{03} a_{20}-a_{12} b_{02}+2 b_{02} b_{03}+a_{02}^{2}\left(4 a_{20}+b_{11}\right)+a_{02}\left(-b_{02}\left(a_{11}+4 b_{02}\right)+b_{12}\right)+b_{13} .
\end{aligned}
$$

We perform a near-identity smooth change of coordinates,

$$
\begin{aligned}
& x=u-\left(\frac{1}{3} d_{21}+\frac{1}{6} e_{12}\right) u^{3}-\left(\frac{1}{2} d_{12}+\frac{1}{2} e_{03}\right) u^{2} v \\
& y=v+d_{30} u^{3}+d_{03} v^{3}-\frac{1}{2} e_{12} u^{2} v-e_{03} u v^{2} .
\end{aligned}
$$

Then system (3.6) becomes

$$
\left\{\begin{aligned}
\frac{d x}{d t}= & y+d_{40} x^{4}+d_{04} y^{4}+g_{31} x^{3} y+d_{13} x y^{3}+d_{22} x^{2} y^{2}+\mathcal{O}\left(|(x, y)|^{5}\right) \\
\frac{d y}{d t}= & b_{20} x^{2}+e_{30} x^{3}+f_{40} x^{4}+y\left(f_{21} x^{2}+f_{31} x^{3}\right)+f_{13} x y^{3}+e_{04} y^{4} \\
& +f_{22} x^{2} y^{2}+\mathcal{O}\left(|(x, y)|^{5}\right)
\end{aligned}\right.
$$

where

$$
\begin{aligned}
& g_{31}=\left(d_{31}-\frac{e_{11}}{2}\left(d_{12}+e_{03}\right)\right), \quad f_{21}=3 d_{30}+e_{21}, \quad f_{40}=\frac{1}{6}\left(b_{20}\left(4 d_{21}-e_{12}\right)+6 e_{40}\right), \\
& f_{31}=b_{20} d_{12}-b_{20} e_{03}+e_{31}, \quad f_{13}=e_{13}, \quad f_{22}=3 b_{20} d_{03}+e_{22} .
\end{aligned}
$$

In order to kill the non-resonant cubic terms of system (3.7), we let

$$
\begin{aligned}
u= & x+\frac{1}{12}\left(d_{04}+d_{13}+e_{04}+f_{13}-f_{22}-3 g_{31}\right) x^{4}+d_{04} y^{4} \\
& +\frac{1}{6}\left(-2 d_{22}+d_{04}+d_{13}+e_{04}+f_{13}\right) x^{3} y \\
v= & y+d_{40} x^{4}+\frac{1}{3}\left(d_{04}+d_{13}+e_{04}+f_{13}-f_{22}\right) x^{3} y \\
& +\left(d_{04}+d_{13}\right) x y^{3}-\frac{1}{2}\left(d_{04}+d_{13}+e_{04}+f_{13}\right) x^{2} y^{2}
\end{aligned}
$$


system (3.7) becomes

$$
\left\{\begin{array}{l}
\frac{d u}{d t}=v+\mathcal{O}\left(|(u, v)|^{5}\right), \\
\frac{d v}{d t}=b_{20} u^{2}+e_{30} u^{3}+f_{40} u^{4}+\left(f_{21} u^{2}+\left(4 d_{40}+f_{31}\right) u^{3}\right) v+\mathcal{O}\left(|(u, v)|^{5}\right) .
\end{array}\right.
$$

Finally, we set $x=b_{20} u, y=b_{20}\left(v+\mathcal{O}\left(|(u, v)|^{5}\right)\right)$, system (3.8) becomes

$$
\left\{\begin{array}{l}
\frac{d x}{d t}=y, \\
\frac{d y}{d t}=x^{2}+\frac{e_{30}}{b_{20}^{2}} x^{3}+\frac{f_{40}}{b_{20}^{3}} x^{4}+y\left(\frac{f_{21}}{b_{20}^{2}} x^{2}+\frac{4 d_{40}+f_{31}}{b_{20}^{3}} x^{3}\right)+\mathcal{O}\left(|(x, y)|^{5}\right) .
\end{array}\right.
$$

According to Lemma 3.8 the above system is equivalent to the system

$$
\left\{\begin{array}{l}
\frac{d x}{d t}=y \\
\frac{d y}{d t}=x^{2}+G x^{3} y+\mathcal{O}\left(|(x, y)|^{3}\right)
\end{array}\right.
$$

where $G=\frac{b_{20}\left(4 d_{40}+f_{31}\right)-e_{30} f_{21}}{b_{20}^{4}}$. Computing the coefficients $b_{20}, d_{40}, f_{31}, e_{30}, f_{21}$ with the condition $(a, d, c, \Lambda, \sigma)=\left(a_{0}, d_{0}, c_{0}, \Lambda_{0}, \sigma_{0}\right)$ and straightforward calculation lead to $G=$ $-1.061119 \times 10^{6}<0$. Thus, $E_{1}$ is a cusp type of Bogdanov-Takens singularity with codimension 3 .

Remark 3.12 The authors in $[13,18]$ proved their epidemic model with saturated incidence rate undergoes a Bogdanov-Takens bifurcation of codimension 2. When we consider the incidence of a combination of the saturated incidence rate and a non-monotonic incidence, the codimension of Bogdanov-Takens bifurcation can grow up to 3 .

Remark 3.13 Xiao and Ruan (see [11]) showed that either the number of infective individuals tends to zero as time evolves or the disease persists. The authors in $[13,18]$ proved that their epidemic model with saturated incidence rate undergoes a Bogdanov-Takens bifurcation of codimension 2. When we consider the incidence of a combination of the saturated incidence rate and a non-monotonic incidence, the codimension of BogdanovTakens bifurcation can grow up to 3 .

\section{Conclusions}

In this paper, by combining qualitative and bifurcation analyses we study an SIS epidemic model with the incidence rate $\frac{a I^{2}}{c+I^{2}}+\frac{b I}{c+I^{2}}$, which is a combination of the saturated incidence rate studied in $[13,18]$, describing the inhibition effect from the behavioral change and the non-monotonic incidence studied by Ruan in [11], interpreting the "psychological" effect. In Sect. 2, we give a full-scale analysis for the types and stability of the equilibria $E_{i}(i=0,1,2,3,4,5)$. We prove that for system (2.1) there can occur backward bifurcation and the backward bifurcation will disappear if $a=0$. At equilibrium $E_{i}(i=3,5)$, a degenerate Hopf bifurcation arises under certain conditions. When the critical condition $\Psi_{i}$ $(i=3,5)$ satisfied, we calculate the Liapunov value of the weak focus and obtain the maximal multiplicity of the weak focus is two, indicating that there exist at most two limit cycles around $E_{i}(i=3,5)$. In Fig. 6 and Fig. 8, we give the phase portraits corresponding to equilibrium $E_{3}$ and $E_{5}$ exhibiting a unique limit cycle and adding a new limit cycle after 
a small perturbation of the parameters $\Lambda$ and $c$. In Sect. 3.3, we proved that the model exhibits Bogdanov-Takens bifurcation of codimension 2 and codimension 3, under certain conditions. If the parameter $a=0$, the model can just have a Bogdanov-Takens bifurcation of codimension 2, shown in [13].

In reality, we show that the model exhibits multi-stable states. This interesting phenomenon indicates that the initial states of an epidemic can determine the final states of an epidemic to go extinct or not. Moreover, the periodical oscillations signify that the trend of the disease may be affected by the behavior of the susceptible and the effect of psychology of the disease.

\author{
Acknowledgements \\ The authors are grateful to both reviewers for their helpful suggestions and comments. \\ Funding \\ This work was supported by the National Natural Science Foundation of China [11771373, 11001235].
}

Availability of data and materials

Not applicable.

Competing interests

The authors declare that they have no competing interests.

Authors' contributions

The authors have made equal contributions. All authors read and approved the manuscript.

\title{
Author details
}

${ }^{1}$ School of Mathematics and Statistics, Central China Normal University, Wuhan, China. ${ }^{2}$ College of Mathematics and System Sciences, Xinjiang University, Urumqi, China.

\section{Publisher's Note}

Springer Nature remains neutral with regard to jurisdictional claims in published maps and institutional affiliations.

Received: 28 July 2018 Accepted: 15 January 2019 Published online: 29 January 2019

\section{References}

1. Anderson, R., May, R.: Infectious Diseases of Humans: Dynamics and Control. Oxford University Press, Oxford (1992)

2. Driessche, P., Watmough, J.: A simple SIS epidemic model with a backward bifurcation. J. Math. Biol. 40, 525-540 (2000)

3. Hu, Z., Teng, Z., Jia, C., Zhang, C., Zhang, L.: Dynamical analysis and chaos control of a discrete SIS epidemic model. Adv. Differ. Equ. 2014, 58 (2014)

4. Hu, Z., Teng, Z., Zhang, L.: Stability and bifurcation analysis in a discrete SIR epidemic model. Math. Comput. Simul. 97, 80-93 (2014)

5. Hu, Z., Teng, Z., Jiang, H.: Stability analysis in a class of discrete SIRS epidemic models. Nonlinear Anal., Real World Appl. 13, 2017-2033 (2012)

6. Liu, W., Levin, S., Iwasa, Y.: Influence of nonlinear incidence rate upon the behavior of SIRS epidemiological models. J. Math. Biol. 23, 187-204 (1986)

7. Hethcote, H., Driessche, P.: Some epidemiological models with nonlinear incidence. J. Math. Biol. 29, $271-287$ (1991)

8. Moghadas, S.: Analysis of an epidemic model with bistable equilibria using the Poincaré index. Appl. Math. Comput. $149,689-702$ (2004)

9. Alexander, M., Moghadas, S.: Periodicity in an epidemic model with a generalized non-linear incidence. Math. Biosci. $189,75-96(2004)$

10. Alexander, M., Moghadas, S.: Bifurcation analysis of an SIRS epidemic model with generalized incidence. SIAM J. Appl. Math. 65, 1794-1816 (2005)

11. Xiao, D., Ruan, S.: Global analysis of an epidemic model with nonmonotone incidence rate. Math. Biosci. 20, 419-429 (2007)

12. Leung, G., et al.: The impact of community psychological response on outbreak control for severe acute respiratory syndrome in Hong Kong. J. Epidemiol. Community Health 57, 857 (2003)

13. Li, J., Zhao, Y., Zhu, H.: Bifurcation of an SIS model with nonlinear contact rate. J. Math. Anal. Appl. 432, 1119-1138 (2015)

14. Zhang, Z., Ding, T., Huang, W., Dong, Z.: Qualitative Theory of Differential Equations. Am. Math. Soc., Province (1992)

15. Arnold, V.: Geometrical Methods in the Theory of Ordinary Differential Equations. Springer, New York (1983)

16. Dushoff, J., Huang, W., Castillo-Chavez, C.: Backwards bifurcations and catastrophe in simple models of fatal diseases. J. Math. Biol. 36, 227-248 (1998)

17. Guckenheimer, J., Holmes, P.: Nonlinear Oscillations, Dynamical System, and Bifurcation of Vector Field. Springer, New York (1996) 
18. Ruan, S., Wang, W.: Dynamical behavior of an epidemic model with a nonlinear incidence rate. J. Differ. Equ. 188, 135-163 (2003)

19. Perko, L.: Differential Equations and Dynamical Systems, 2nd edn. Texts in Applied Mathematics, vol. 7. Springer, New York (1996)

20. Lamontagne, Y., Coutu, C., Rousseau, C.: Bifurcation analysis of a predator-prey system with generalized Holling type III functional response. J. Dyn. Differ. Equ. 20, 535-571 (2008)

Submit your manuscript to a SpringerOpen ${ }^{\odot}$ journal and benefit from:

- Convenient online submission

Rigorous peer review

Open access: articles freely available online

- High visibility within the field

- Retaining the copyright to your article

Submit your next manuscript at $\boldsymbol{\nabla}$ springeropen.com 